\title{
EchoGéo
}

33 | 2015

Activisme, participation, contestation : la place des habitants dans les processus de patrimonialisation en périphéries urbaines

\section{La place des habitants dans la patrimonialisation conflictuelle du logement social}

Études de cas croisés à Plaine Commune

\section{Géraldine Djament-Tran}

\section{(2) OpenEdition \\ 1 Journals}

\section{Electronic version}

URL: https://journals.openedition.org/echogeo/14349

DOI: $10.4000 /$ echogeo. 14349

ISSN: 1963-1197

Publisher

Pôle de recherche pour l'organisation et la diffusion de l'information géographique (CNRS UMR 8586)

\section{Electronic reference}

Géraldine Djament-Tran, "La place des habitants dans la patrimonialisation conflictuelle du logement social", EchoGéo [Online], 33 | 2015, Online since 30 September 2015, connection on 10 August 2021. URL: http://journals.openedition.org/echogeo/14349 ; DOI: https://doi.org/10.4000/echogeo.14349

This text was automatically generated on 10 August 2021

EchoGéo est mis à disposition selon les termes de la licence Creative Commons Attribution - Pas d'Utilisation Commerciale - Pas de Modification 4.0 International (CC BY-NC-ND) 


\title{
La place des habitants dans la patrimonialisation conflictuelle $\mathrm{du}$ logement social
}

Études de cas croisés à Plaine Commune

\author{
Géraldine Djament-Tran
}

Patrimoine alternatif donnant de la valeur à ce qui en a peu dans la société, le patrimoine $\mathrm{du}$ logement social semble relever de la patrimonialisation par appropriation, bottom up et non institutionnelle ${ }^{1}$, par opposition à la patrimonialisation par désignation (Rautenberg, 2003), top down, typique de la fabrique nationale institutionnelle du patrimoine (Heinich, 2009). La rupture qu'il accomplit avec le traditionnel "classement de classe" des Monuments Historiques (Aguilard, 1982) semble porteuse d'un empowernment patrimonial des habitants, comme dans le cas du Musée urbain Tony Garnier à Lyon (Chenevez, 2004). Cependant, au moment même où émerge cette catégorie récente, fruit de l'élargissement chronologique, géographique, typologique et conceptuel de la notion de patrimoine, le logement social se trouve dévalorisé par une idéologie dominante privilégiant l'accession à la propriété et stigmatisant notamment les grands ensembles, tandis que ses habitants connaissent une crise liée à la métropolisation. Le passage de la ségrégation associée à la ségrégation dissociée les confronte au chômage de masse. L'augmentation des inégalités et l'évolution vers la "ville à trois vitesses » impliquent le départ des habitants les plus favorisés et débouchent sur une "nouvelle question urbaine " (Donzelot, Jaillet, 1999) marquée par des émeutes. La décomposition post-industrielle des territoires du communisme (Bellanger, Mischi, 2013) participe de la désaffiliation sociale et politique des catégories populaires (Castel, 1991 ; Bacqué, Sintomer, 2001) peu propice aux mobilisations en général, patrimoniales en particulier. Face aux tendances à la ghettoïsation des quartiers populaires, qui suscitent de nombreux débats (Wacquant, 2006 ; Lapeyronnie, 2008), quel rôle jouent les habitants dans le processus conflictuel de patrimonialisation du logement social? Loin d'être d'anecdotique, la question représente un observatoire décalé des nouveaux mouvements sociaux urbains, qui évoluent vers une « diversification des objets de mobilisation » et « un déplacement 
vers les questions environnementales, d'espace public et d'art dans la ville » (Douay, 2012), mais aussi de la crise urbaine et des "métamorphoses de la question sociale " (Castel, 1995).

2 Nous interrogerons la façon dont la pauvreté et les problèmes sociaux deviennent un obstacle à et/ou une cible de la patrimonialisation à partir du terrain de Plaine Commune, communauté d'agglomération de banlieue nord de Paris, observatoire privilégié à plusieurs titres. Lieu d'expérimentation pour la construction de logement social depuis la fin du XIX siècle (Pouvreau, 2008), cette ancienne banlieue rouge (Bacqué, Fol, 1997) qui a célébré en 2012 le centième anniversaire du logement social (association Toit et moi, 2015) présente, comme le département tout entier (Pouvreau, 2003), une proportion de logement social particulièrement élevée. Bien que de plus en plus intégré économiquement à la métropole parisienne, ce territoire post-industriel, avec son précariat (Castel, 2009) qui tend à remplacer le prolétariat, et de nombreuses populations issues de l'immigration, souffre d'une marginalisation sociale (Lebeau, 2007) typique des « espaces ciseaux » (Albecker, 2014).

Tableau 1 - Les habitants de Plaine Commune face à la crise des quartiers populaires

\begin{tabular}{|l|l|l|l|l|l|}
\hline Commune & Villetaneuse & Pierrefitte & Stains & $\begin{array}{l}\text { Saint- } \\
\text { Denis }\end{array}$ & $\begin{array}{l}\text { Plaine } \\
\text { Commune }\end{array}$ \\
\hline Taux de chomage & $24,3 \%$ & $20,2 \%$ & $22,1 \%$ & $23,7 \%$ & $22,3 \%$ \\
\hline Taux de pauvreté & $32,3 \%$ & $36,3 \%$ & $34,4 \%$ & $35,4 \%$ & $35,4 \%$ \\
\hline $\begin{array}{l}\text { Médiane du revenu disponible par } \\
\text { unité de consommation }\end{array}$ & $14574,3 €$ & $14140 €$ & $14358,8 €$ & $14385,7 €$ & $14315 €$ \\
\hline Ménages fiscaux imposés & $50,5 \%$ & $50,8 \%$ & $49,1 \%$ & $52 \%$ & $52,1 \%$ \\
\hline
\end{tabular}

Les chiffres sont à l'échelle des municipalités ou de l'intercommunalité et non des quartiers étudiés. Source : INSEE 2012.

3 L'intercommunalité tente de promouvoir le patrimoine de banlieue (Jacquot, GravariBarbas, Fagnoni, 2013) comme palliatif à la paupérisation. Notre analyse comparative se fonde sur une série d'entretiens semi-directifs avec les acteurs locaux (cf. liste en annexe). 
Illustration 1 - Le patrimoine du logement social à Plaine Commune

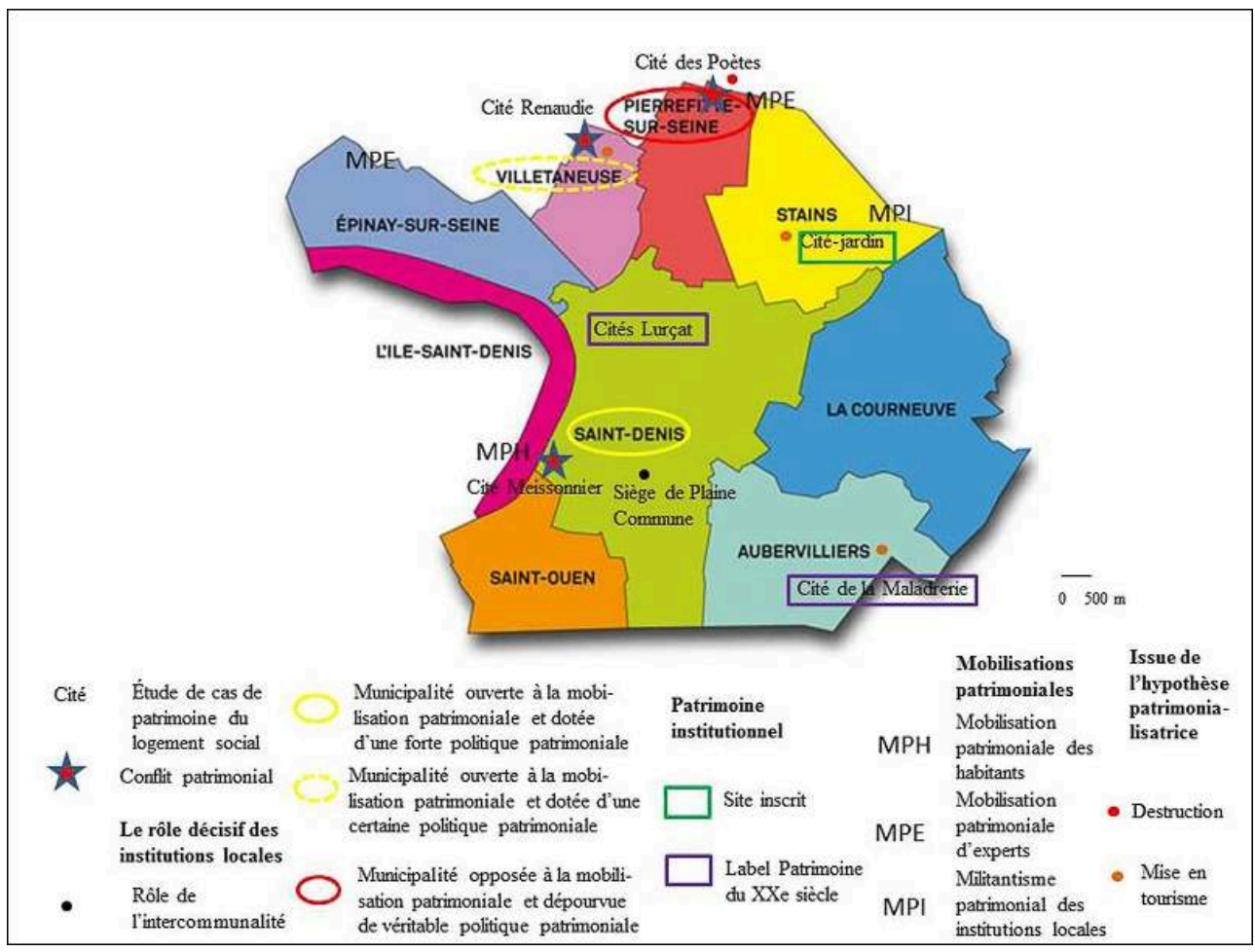

Source : fond de la Mairie d'Epinay sur Seine ; réalisation : G. Djament-Tran.

\section{La mobilisation patrimoniale des habitants du logement social rare et insuffisante à la patrimonialisation}

\section{Un cas exceptionnel supposant l'ancrage des habitants}

La cité Meissonnier de Saint-Denis, ensemble de logements ouvriers ressemblant à un coron, inaugurée en 1913 dans une ancienne usine de teinturerie à proximité du carrefour Pleyel présente le cas exceptionnel où une association d'habitants Meissonnier La Garance, créée en 2000 - joue un rôle moteur dans la patrimonialisation. Le projet municipal et départemental de destruction de la cité au profit de la construction d'un foyer d'immigrés puis d'une route désengorgeant le quartier Pleyel se heurte à la mobilisation de quelques ouvriers locataires. Cette mobilisation s'explique par leur enracinement dans la cité (la famille du président de l'association y est installée depuis 1944) et par leur engagement communiste. Elle est conduite par la famille de la concierge, d'origine bretonne - Saint-Denis ayant accueilli une importante immigration bretonne au début $\mathrm{du} \mathrm{XX}^{\mathrm{e}} \mathrm{s}$. - qui défend son lieu d'habitat depuis plusieurs générations, son cadre de vie spacieux et convivial, photographié par Doisneau On passe donc d'un attachement au patrimoine familial à une mobilisation patrimoniale qui dure une décennie, du fait des difficultés de la mairie à trouver un montage financier satisfaisant pour la réhabilitation de la cité. Les habitants lancent des pétitions, organisent des réunions, prennent contact avec les élus, font visiter la cité et invitent des artistes lors des Journées du Patrimoine. Une fresque réalisée 
conjointement par une plasticienne connue par l'association patrimoniale Lucerna, par les habitants et les enfants du centre de loisirs Pleyel rend hommage à la mémoire ouvrière.

Illustration 2 - La cité Meissonnier

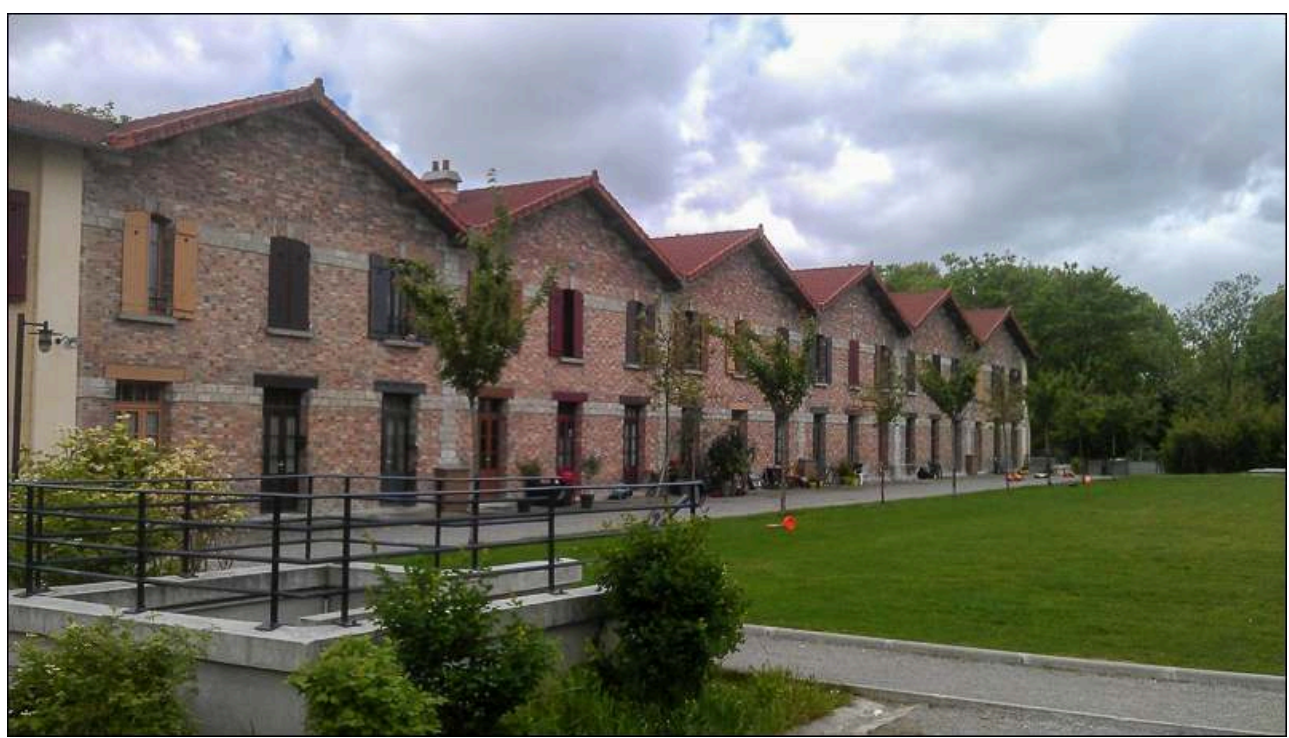

Auteur : G. Djament-Tran.

\section{La connexion décisive entre la mobilisation habitante et un écosystème patrimonial local}

Le succès de cette mobilisation habitante qui débouche sur la réhabilitation de la cité (2008-2010) s'explique par sa connexion avec un écosystème patrimonial local. Un élu municipal communiste et militant patrimonial (premier président de Franciade, association montée par l'Unité d'archéologie de Saint-Denis proche de l'économie sociale et solidaire qui produit des objets dérivés du patrimoine) aide au montage de l'association. Les habitants bénéficient également de l'appui de deux autres élus municipaux communistes, P. Braouezec et un de ses proches, et s'y investit. En outre, la mobilisation habitante se trouve relayée par les institutions patrimoniales nationales, départementales et municipales. L'Architecte des Bâtiments de France de Seine SaintDenis sanctionne la valeur patrimoniale de la cité et prescrit la conservation à l'identique de l'extérieur des bâtiments. Il rend possible son sauvetage en contactant un promoteur à même d'en assurer la réhabilitation, traditionnellement spécialisé dans les Monuments Historiques: Histoire et Patrimoine. Le service Patrimoine du Conseil général intervient également. La mobilisation patrimoniale habitante forme progressivement système avec la politique patrimoniale municipale. L'Unité d'archéologie de Saint-Denis réalise de fructueuses fouilles préventives avant les travaux du parking de la cité et étaye son dossier patrimonial. La valorisation du patrimoine de l'immigration bretonne dans la cité lors de Journées du Patrimoine entre en résonnance avec l'exposition montée sur le sujet par P. Douzenel, photographe de Saint-Denis. La concierge de la cité est invitée à réaliser un photo-montage sur sa famille pour l'exposition Douce banlieue en 2004. La cité Meissonnier se trouve aujourd'hui sur le point d'être intégrée au PLU patrimonial de Saint-Denis, sur la base 
du diagnostic patrimonial réalisé par le service Patrimoine du Conseil général. Les échanges au sein du tissu associatif local contribuent enfin à construire dans la durée une mobilisation patrimoniale : l'association Meissonnier trouve des relais à l'Amicale des Bretons de Saint-Denis et auprès des associations patrimoniales Franciade et Lucerna.

\section{La coproduction habitante et institutionnelle du patrimoine.}

Initialement fondé sur une patrimonialisation par appropriation, le cas de la cité Meissonnier débouche sur une coproduction habitante et institutionnelle du patrimoine du logement social, qui se manifeste aussi aux débuts de la mise en patrimoine de la cité-jardin de Stains. Construit par les architectes Gonnot et Albenque entre 1921 et 1933 et peuplé d'ouvriers travaillant dans les usines de Saint-Denis, ce quartier relevant du courant utopique international initié par E. Howard est inscrit à l'inventaire au titre des sites pittoresques (la catégorie patrimoine du logement social n'étant alors pas diffusée) dès 1976. Mais sa véritable mise en patrimoine, au début des années 2000, découle de la conjonction entre une demande habitante et l'action du service Patrimoine du CG de Seine Saint-Denis. L'Amicale des locataires cherche alors un appui face au propriétaire (l'OPHLM de Seine Saint Denis) afin d'obtenir une réhabilitation du quartier, sous-entretenu. Elle sollicite ce service Patrimoine en vue d'un projet d'exposition à destination des habitants. Nous retrouvons ici la proximité entre patrimoine familial et urbain, ainsi que le rôle clé joué par l'ancrage des habitants dans leur mobilisation. L'Office HLM a en effet dans les années 1960 donné aux locataires un droit de filiation (rare et paradoxal en logement social), si bien qu'un attachement au quartier s'est développé. La mise en place d'un cercle vertueux conduit à un projet de réhabilitation à caractère patrimonial avec des fonds européens et à l'implication de Plaine Commune.

7 Si la seule mobilisation des habitants n'apparaît pas suffisante, elle ne se révèle cependant pas non plus nécessaire à la patrimonialisation du logement social, comme le montre le cas de la cité Renaudie de Villetaneuse.

\section{L'indifférence fréquente des habitants à la patrimonialisation du logement social, observatoire de la désaffiliation dans les quartiers populaires}

8 Dernière opération, en partie posthume (1976-1983), de Jean Renaudie (1925-1981, Prix d'Architecture en 1978), le Vieux Pays constitue une opération de résorption d'habitat insalubre et une partie d'un projet plus vaste de remodelage du centre de Villetaneuse jamais réalisé. Son architecture, dite " proliférante ", en étoile avec terrasses arborées relève d'une utopie de l'habiter. Mais les habitants rencontrent assez rapidement au quotidien des difficultés concrètes liées à des problèmes techniques (défauts d'étanchéité, coût du chauffage) qui rendent la gestion de la cité complexe et onéreuse. En outre, la cité Renaudie devient un logement ultra-social : un de ses bailleurs, ICF La Sablière, qui loge principalement des cheminots, y regroupe, à part des cheminots, des populations défavorisées lui permettant de satisfaire aux quotas réglementaires du logement social. La situation sociale se dégrade, marquée par des intrusions dans les cœurs d'ilots initialement conçus pour assurer la convivialité locale. Cette situation 
conduit ICF à déposer un dossier de démolition partielle, au début des années 2000, au titre des périmètres dérogatoires pouvant bénéficier des crédits ANRU. Une partie de la cité est murée et vidée de ses habitants.

Si une mobilisation patrimoniale émerge et empêche la démolition, elle n'est pas imputable aux habitants. En effet ceux-ci appartiennent à des catégories très populaires touchées par la désaffiliation (contrairement à Meissonnier où les habitants, d'origine populaire, sont affiliés à la société salariale et à la ville ouvrière) et ils ne sont pas organisés collectivement (il n'existe pas d'Amicale de locataires; seule une association a été constituée récemment pour lutter contre des dealers investissant des logements squattés). La mobilisation est au contraire menée par des architectes : Serge Renaudie, fils de l'architecte, écrit fin 2002 au Ministre de la culture et lance une pétition sur internet en 2003. Son succès tient beaucoup à la réactivité des institutions patrimoniales. Le service patrimoine du CG, avec la DRAC d'Ile de France, interpelle dans le cadre du protocole de décentralisation le ministère de la Culture, en demandant une instance de classement. Le ministère lui préfère la mission d'enquête, co-réalisée avec le ministère de l'Equipement, dont le rapport recommande en 2004 la conservation de cet îlot représentatif des utopies du $\mathrm{XX}^{\mathrm{e}}$ siècle, tout en préconisant son adaptation au vu des dysfonctionnements. La mairie communiste refuse alors de signer le permis de démolir.

Aujourd'hui, la cité Renaudie a été non seulement conservée mais rénovée, de concert avec l'ABF et S. Renaudie. Sa patrimonialisation dans le cadre de la politique municipale de valorisation des "utopies réalisées" et de la politique touristique de Plaine Commune a permis la pose d'une signalétique touristique, l'organisation de balades urbaines. Le patrimoine du logement social fait même l'objet d'un projet de protection au titre du PLU.

11 Cette patrimonialisation ne nous semble relever ni de la patrimonialisation par appropriation (déficiente) ni de la patrimonialisation par désignation (absente), mais d'une troisième logique, que nous proposons de qualifier de patrimonialisation par expertise : ce ne sont ni les habitants ni la procédure institutionnelle traditionnelle qui président à la reconnaissance de la valeur patrimoniale, mais des spécialistes. 
Illustration 3 - La cité Renaudie de Villetaneuse

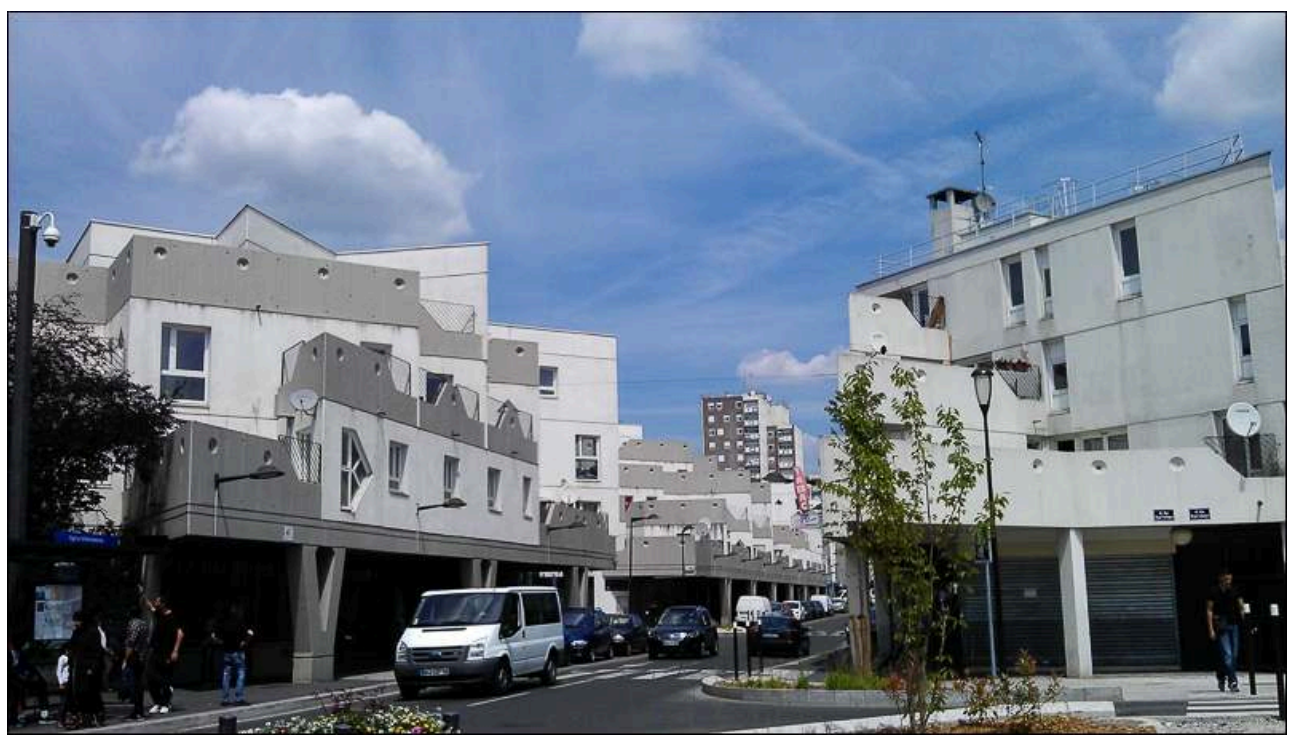

Auteur : G. Djament-Tran.

Illustration 4 - La mise en patrimoine de la cité Renaudie de Villetaneuse

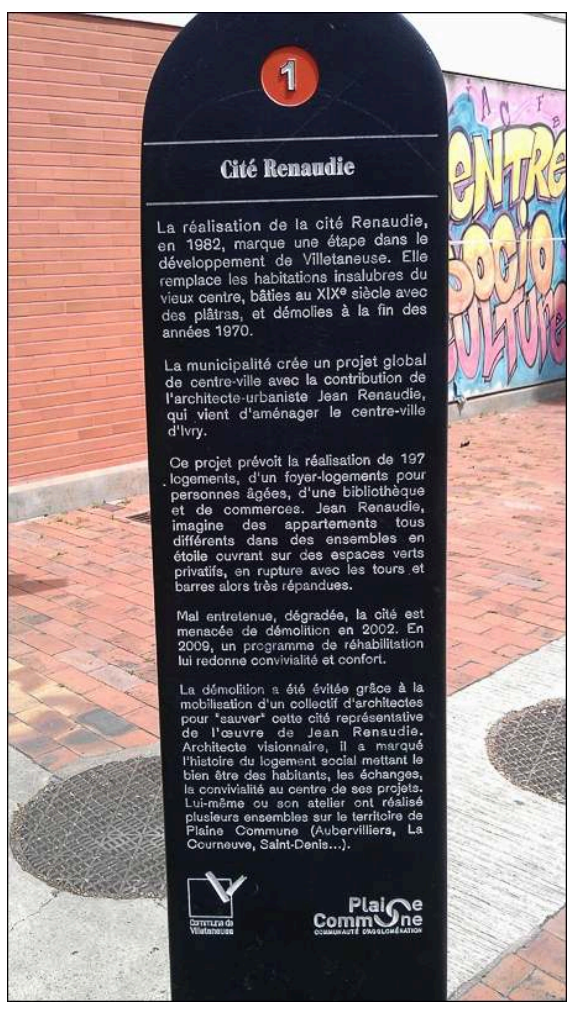

Auteur : G. Djament-Tran 


\section{Un double risque dans le rapport des habitants au patrimoine du logement social}

12 Le cas de la cité Renaudie révèle les problèmes sociaux auxquels se heurte la patrimonialisation du logement social. Une montée en généralité peut s'effectuer sur la base du rapport d'inspection qui concluait qu'« une occupation sociale ainsi paupérisée n'était pas propice à la valorisation de l'habitat conçu par Jean Renaudie », soulignant que «la réussite dans la durée implique (...) que les habitants soient mis en situation d'apprécier l'habitat qui leur est proposé $»^{2}$.

\section{Le risque de mobilisation anti-patrimoniale des habitants victimes d'une grave dégradation de l'habiter}

13 Ces remarques connaissent un écho particulier au vu du cas de la cité des Poètes à Pierrefitte sur Seine, marqué non seulement par une indifférence patrimoniale des habitants, mais par une mobilisation anti-patrimoniale d'une partie d'entre eux, dans un contexte de grave crise de l'habiter.

Cette crise s'enracine dans la difficile genèse du quartier, construit au nord de la commune au bord de la Nationale 1, dans un contexte de rapide croissance démographique, par la municipalité à l'époque communiste et la SODEDAT 93, qui font appel à de jeunes architectes disciples de J. Renaudie: les frères Euvremer pour l'ensemble Brassens (1983) et Jeronimo Pedron-Lopez pour l'ensemble Desnos (1986). Dès le départ, problèmes financiers et nombreuses malfaçons forment un cercle vicieux. Si, au départ, la cité accueille une partie de classes moyennes, elle concentre rapidement, conformément à une trajectoire classique, des populations beaucoup plus fragilisées, sans ancrage territorial, en particulier de nombreux primo-arrivants d'Afrique subsaharienne à la suite du relogement des habitants de la cité de transit de l'avenue Lénine. Avec la crise économique, la «lutte pour ce quartier " monument » " menée par les premiers habitants ${ }^{3}$ fait place aux difficultés quotidiennes (Guillerm, 2006, p. 204). Au cours des années 1990, le Centre social et culturel initie une revalorisation du quartier, classé zone urbaine sensible dès 1996. Mais au début des années 2000 , il sombre dans la délinquance et connaît de nombreuses dégradations. La convivialité prévue se transforme en entre-soi des habitants, qui se barricadent dans leurs logements. Les forces de l'ordre peinent à accéder à la cité piétonne.

Dans cette situation sociale très difficile ${ }^{4}$, une première étude prônait la réhabilitation de la cité, mais la grosse enveloppe financière associée par l'ANRU à la démolition des ensembles Desnos et Brassens convainc la municipalité et Plaine Commune de retenir cette option. Comme à Villetaneuse, la mobilisation patrimoniale est menée par des architectes, renaudiens en majorité ${ }^{5}$ ou non ${ }^{6}$. Une première pétition, signée par 130 professionnels, est déposée en juin 2005. Une seconde pétition, «Non à la démolition des 450 HLM expérimentaux du quartier des Poètes ! ", lancée début 2010, regroupe des architectes, mais aussi des élus à la sensibilité patrimoniale, des enseignants, des plasticiens. Docomomo (Association Française pour la DOcumentation et la Conservation des édifices, sites et ensembles urbains du MOuvement Moderne), section française de Docomomo International créée en 1991, intervient auprès du ministère de la Culture. Elle réclame une procédure Monuments Historiques et dépose en octobre 2009 une requête en annulation du permis de démolir auprès du Tribunal 
Administratif de Montreuil au nom de la valeur patrimoniale et d'une contribution pionnière au développement durable. À côté de cette mobilisation patrimoniale, soutenue par l'association Paris historique, une mobilisation anti-démolition fondée sur un argumentaire social se manifeste sur un mode mineur (Veschambre, 2013). La Confédération nationale du logement, le Comité Actions Logement et l'Union Sociale de l'Habitat s'inquiètent «du manque de garantie quant à l'augmentation de loyers » et dénoncent «le manque de prise en compte de l'avis des habitants» (Guillerm, mém. cit., p. 206).

Mais contrairement au cas Renaudie, le cas des Poètes présente une mobilisation habitante anti-patrimoniale. En effet, fin 2009, l'Association de défense du projet de rénovation urbaine du quartier des poètes de la ville de Pierrefitte-sur-Seine, proche de la municipalité désormais socialiste de la ville, est créée, présidée par un éducateur spécialisé militant associatif originaire des Comores. Elle regroupe bientôt 150 adhérents $^{7}$. Soulignons cependant que cette mobilisation des habitants en faveur de la démolition n'intervient que lorsque celle-ci est déjà entamée, et difficilement réversible. Le 30 novembre 2009, un référé du Tribunal Administratif suspend la démolition en cours, ce qui entraîne une explosion du trafic de drogue sur le chantier à l'arrêt et nécessite un arrêté de péril de la mairie. Une pétition pour la reprise des travaux de démolition est alors lancée par la mairie, signée par plus de 2000 Pierrefittois $^{8}$ et remise au ministère du Logement le 18 janvier 2010.

La position des habitants, en majorité passifs et non convaincus de la valeur patrimoniale de leur logement, semble en fait avoir été plus complexe que ne le laisse supposer une partie de la presse, réduisant le conflit à un affrontement entre " architectes et résidents " . Les habitants semblent surtout avoir été favorables à une revalorisation du quartier, qu'il s'agisse d'une réhabilitation de l'existant, en faveur de laquelle 800 résidents ont signé en 2004 une pétition ${ }^{10}$, ou d'une démolitionreconstruction, approuvée à l'unanimité (après présentation de la municipalité en sa faveur) en novembre 2005 par 150 habitants réunis lors d'un Conseil de quartier (Guillerm, mém. cit.). Cette option représente alors une opportunité pour quitter le quartier.

En outre, si en conseil de quartier, une majorité d'habitants hue les interventions propatrimonialisation d'architectes et dénonce leur méconnaissance des conditions locales de l'habiter, une minorité, appartenant aux classes moyennes et assez anciennement ancrée dans la cité (y compris des immigrés installés de relativement longue date), se mobilise pour son cadre de vie. Le recours en justice piloté par Dococomo est aussi déposé par une habitante de Desnos et une de Brassens.

Le conflit portant sur la valeur patrimoniale du site oppose surtout les architectes à la municipalité et l'ANRU. L'actif lobbying de la municipalité en faveur de la démolition oppose à la logique patrimoniale le supposé lien causal cher à l'ANRU entre l'architecture et les problèmes sociaux. Le tribunal administratif de Montreuil juge le 25 février 2010 recevable sa dénonciation de la crise de l'habiter comme justification de la démolition, se retranchant lui aussi derrière une conception du patrimoine limité au patrimoine institutionnel, voire aux Monuments Historiques et à leurs abords ${ }^{11}$. Quant au service rénovation urbaine de Plaine Commune, bien que gêné par la question de la valeur architecturale du site, il considère que la démolition, finalisée en 2012, (et surtout? - la dispersion des habitants ${ }^{12}$ ), constituait le plus sûr remède contre la somme de problèmes agrégés dans la cité. 
Les deux cas des Poètes et de Renaudie directement comparables et liés en une mobilisation patrimoniale partiellement commune, trouvent cependant une issue opposée. Cela ne s'explique pas tant finalement par la différence d'attitude des habitants que par une série de conditions défavorables à la conservation à Pierrefitte. S'y cumulent en effet un manque de notoriété de l'architecte, un enclavement municipal, une mobilisation plus limitée au cercle des architectes proliférants et surtout trop tardive, une municipalité en conflit depuis le chantier avec l'architecte et partisane jusque boutiste de la démolition, la non intervention du Conseil général et du ministère de la Culture, démobilisés face à un combat semblant perdu d'avance, face au lobbying de la municipalité et de la préfecture et face à la grave crise sociale. Surtout, la dégradation majeure de l'habiter semble avoir franchi un seuil rédhibitoire à la patrimonialisation à la cité des Poètes.

Illustration 5 - La rénovation urbaine de la cité des Poètes

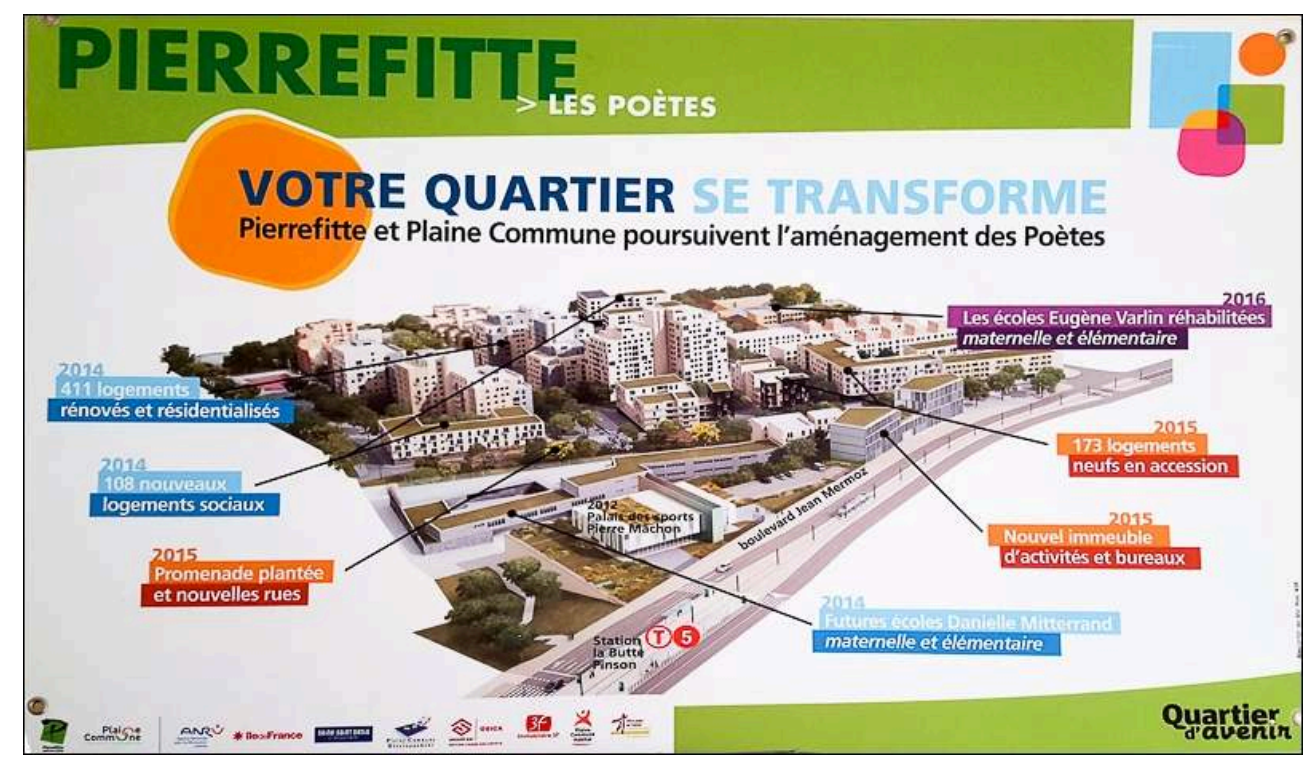

Photographie d'une affiche de Plaine Commune par G. Djament-Tran.

\section{Le risque d'une reconnaissance patrimoniale synonyme de changement social des habitants}

Si la notion paradoxale de patrimoine du logement social peut se heurter à une remise en cause de son caractère patrimonial, la crise de l'habiter conduisant à refuser de transmettre, elle peut également connaître une remise en cause de son caractère social. Alliée objective d'une certaine gentrification (Semmoud, 2005), dans la mesure où elle permet la réhabilitation au lieu de la destruction, la patrimonialisation s'accompagne d'une sortie du logement social. Pire, le changement social des habitants et/ou des propriétaires apparaît comme une condition de la patrimonialisation. À la cité Meissonnier, pour rendre financièrement rentable l'opération de réhabilitation du patrimoine, des logements neufs, petit collectif et maisons de ville, ont été construits et en partie vendus à une clientèle aisée qui y investit. Certains locataires ont également pu devenir propriétaires de leur logement. Les loyers sont devenus élevés, sauf pour les locataires sous le régime de la loi de 1948 qui ont ainsi pu rester sur place. 

l'accession à la propriété. La démolition est évitée par le rachat et la réhabilitation de la partie menacée de la cité par le promoteur dionysien DCF, dont le président, proche de Plaine Commune, sensibilisé à l'architecture de Renaudie, constitue la SCI « Les Jardins Renaudie ». 84 logements sont vendus entre 2010 et 2012, en majorité à des primoaccédants à la propriété originaires du logement social, certains étant sensibilisés à l'architecture en terrasses.

Dans les deux cas, la patrimonialisation-gentrification s'est accompagnée d'une certaine fermeture résidentielle. Les espaces collectifs conçus par Renaudie ont été clôturés pour limiter les problèmes d'intrusion et les accès aux logements complètement modifiés. La cité rebaptisée "villa Meissonnier» a été également « résidentialisée » (close). L'architecture a certes été sauvée, mais le patrimoine (qui n'est donc plus que partiellement patrimoine du logement social, même si l'on reste dans de l'habitat populaire, notamment à Villetaneuse) a été transformé à l'épreuve de la crise sociale.

Illustration 6- La sortie du logement social d'une partie de la cité Renaudie de Villetaneuse

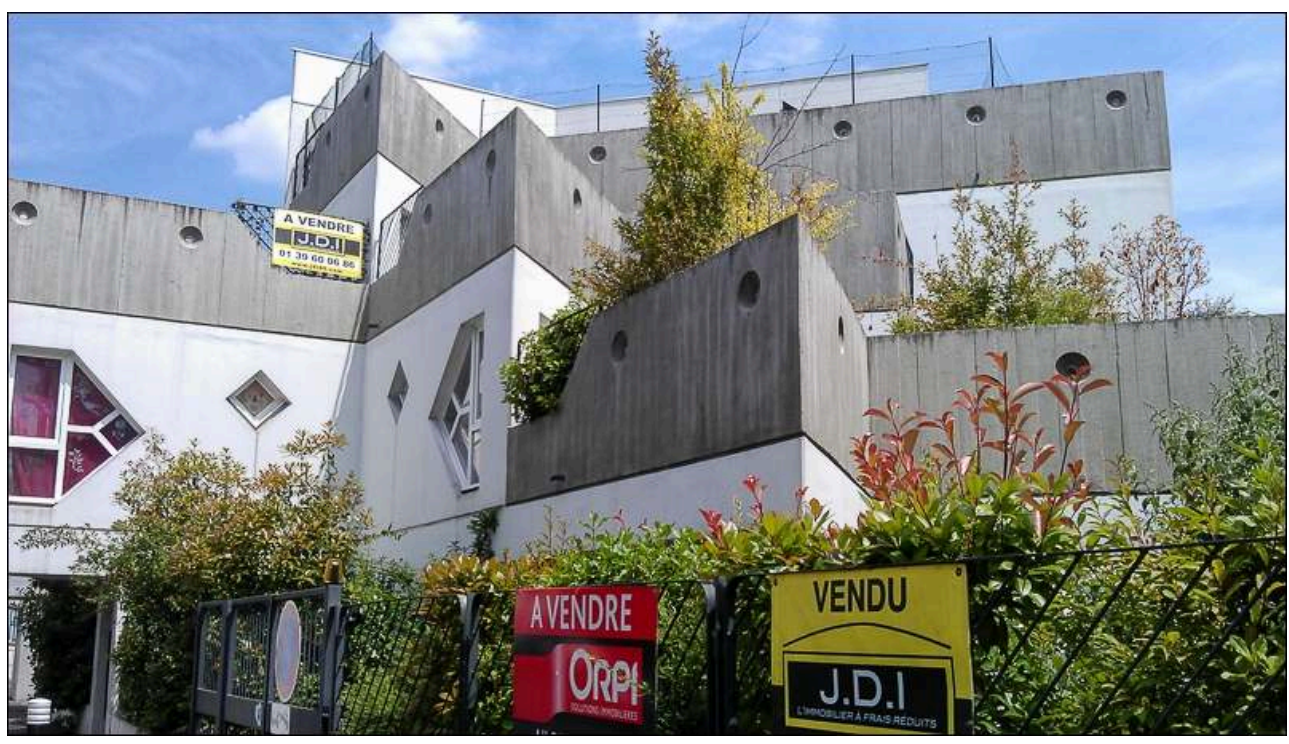

Auteur : G. Djament-Tran.

À Villetaneuse, les nouveaux copropriétaires se heurtent en outre de prime abord, avant l'intervention de médiateurs, à des cambriolages et intrusions. Comme à Meissonnier, où la valorisation patrimoniale menée par les habitants a été stoppée à la suite des bagarres et des vols marquant les Journées du Patrimoine 2007, la cohabitation se révèle difficile entre un patrimoine relativement gentrifié et les quartiers populaires environnants, touchés par la marginalisation. La patrimonialisation participerait-elle ainsi de la tendance à la fragmentation métropolitaine?

Inversement, la patrimonialisation se trouve mobilisée par les pouvoirs publics locaux pour tenter de remédier à la dévalorisation de l'habitat populaire. Le service rénovation urbaine de Plaine Commune mène ainsi dans la partie restée en HLM, de concert avec des associations, une politique pour que les habitants ne subissent pas l'architecture de Renaudie mais se l'approprient. L'aide d'un paysagiste à la plantation 
des terrasses, des interventions de l'Association pour les Equipements sociaux sont sollicitées tandis que les services publics municipaux et intercommunaux requalifient le quartier. L'appropriation des terrasses reste cependant très variable selon les habitants.

\section{Non pas un militantisme des habitants mais un militantisme de l'habiter}

Si les différents conflits étudiés ne permettent pas de conclure à un militantisme patrimonial des habitants à Plaine Commune, en revanche l'intercommunalité apparaît porteuse d'un militantisme de l'habiter. En effet on assiste à l'hybridation des politiques patrimoniales et touristiques, qui relèvent de la direction du développement local, où elles voisinent avec l'économie sociale et solidaire, et des politiques sociales. Cette hybridation est au service d'une tentative de revalorisation du logement social, comme l'illustre le cas emblématique de la cité-jardin de Stains.

Plaine Commune y mène en effet depuis 2004, avec la nomination d'une chargée de valorisation touristique, suivie en 2008 de l'ouverture du local Mémoires de cité-jardin, une active politique patrimoniale (pause de bornes touristiques, organisation de balades urbaines, manifestations culturelles, actions artistiques), fleuron de son schéma touristique et argument pour l'obtention du label Ville d'art et d'histoire obtenu fin 2013. Quelle place occupent les habitants dans cette mise en patrimoine et en tourisme participative?

\section{Les habitants principaux destinataires d'une mise en patrimoine et en tourisme participative}

Si la tension entre patrimonialisation et pauvreté suscite au départ quelques réactions d'hostilité13, le travail d'insertion dans la vie de quartier de la chargée de valorisation touristique permet la participation habitante au projet institutionnel.

Quelques habitants sont coproducteurs de la mise en patrimoine. Les associations locales et/ou l'Amicale des locataires appuient fréquemment les manifestations organisées par la chargée de valorisation touristique. L'association Accueil Banlieues, fondée en 2011 et présente essentiellement sur le territoire de Plaine Commune, loge chez l'habitant à prix modiques des visiteurs et leur fait découvrir les richesses du territoire de banlieue.

Surtout, les habitants sont les principaux destinataires de la politique patrimoniale et « touristique » qui touche en fait majoritairement des Franciliens. Opérations de recueil de mémoire, reportages photographiques, séances de lightpainting ${ }^{14}$, ateliers créatifs (notamment pour la production d'une gamme de produits dérivés en partenariat avec Franciade) les incitent à participer à la mise en patrimoine. Les habitants sont partie prenante de la concertation avec l'ABF et le bailleur autour de la rénovation urbaine (2006-2014). Le soin apporté à la dimension conviviale des manifestations patrimoniales et la fonction de sociabilité assumée par le local Mémoires de cité-jardin ${ }^{15}$ décloisonnent politique de la ville et politique patrimoniale, dont une partie du financement provient d'ailleurs initialement d'un Contrat Urbain de Cohésion Sociale. 


\section{Les limites de la participation habitante}

31 La participation se révèle toutefois limitée et fragile. Seule une minorité d'habitants participe régulièrement aux manifestations culturelles, essentiellement des enfants et des retraités. Les actions patrimoniales s'appuient sur un groupe d'habitants-clés, de l'ordre d'une dizaine de personnes, de catégories sociales populaires et non politisées mais ancrées dans la cité-jardin et sur les structures (para-)scolaires. La mobilisation patrimoniale des habitants apparaît en outre aléatoire, dans la mesure où elle dépend des relations personnelles de la chargée de valorisation touristique. Cependant, certains habitants, ancrés dans la cité-jardin, sont fidélisés par la politique patrimoniale, qui transforme leur rapport à leur habitat, et vient d'être reconnue par le prix Europa Nostra du patrimoine dans la catégorie sensibilisation.

Illustration 7- Mémoires de Cité-jardin,centre du militantisme patrimonial et du tourisme participatif à Stains

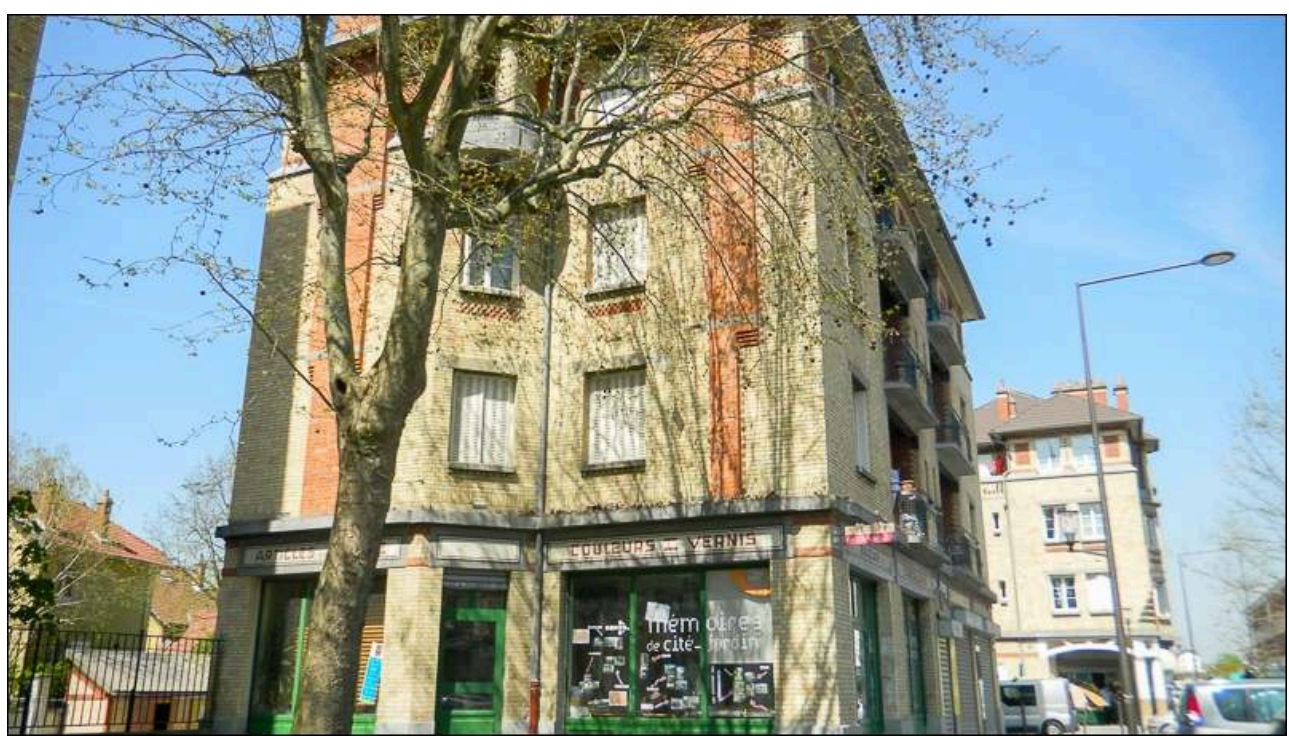

Auteur : G. Djament-Tran.

Ce cas constitue un modèle de la configuration la plus courante sur le territoire : un militantisme patrimonial - expression qui s'inspire de l'application de la notion de militantisme à l'art et à l'architecture (Prévot, Douay, 2012) - des institutions locales. La mise en patrimoine de la banlieue, remède à la crise post-industrielle et à un imaginaire collectif dépréciatif, vise à (re)faire territoire (Auduc, 2012). À Stains, la citéjardin, quartier populaire mais pas insécuritaire, contrairement au quartier voisin de grands ensembles le Clos Saint Lazare, est d'ailleurs devenue une vitrine et un centre.

\section{Conclusion}

Nous avons donc examiné quatre figures du rapport des habitants à la patrimonialisation du logement social :

- la mobilisation patrimoniale,

- la participation à la mise en patrimoine,

- l'indifférence, 
- la mobilisation anti-patrimoniale. la cité-jardin de Stains, à la cité Renaudie de Villetaneuse et à la cité des Poètes de Pierrefitte, tous ces cas se révèlent cependant plus complexes. En effet, tous les habitants ne se mobilisent pas à Meissonnier ni ne participent à la cité-jardin; inversement, le positionnement des habitants est évolutif et partagé aux Poètes. Finalement, l'indifférence relevée à Villetaneuse semble le cas le plus courant. La mobilisation habitante semble en effet dépendre principalement de trois paramètres :

- un paramètre sociologique - le degré de pauvreté et de difficultés sociales étant en général corrélé avec la faiblesse voire l'absence des mobilisations, patrimoniales ou non -,

- un paramètre socio-géographique - l'ancrage territorial des habitants (défini par la relative ancienneté d'habitation dans la cité et par la création d'un sentiment d'appartenance) semble décisif pour la mobilisation -

- et un paramètre politique - leur affiliation politique (dans ce territoire en général communiste) est propice à la mobilisation.

ce contexte, l'issue de la patrimonialisation semble dépendre non pas tant de l'action des habitants que d'un positionnement sur l'échelle des problèmes sociaux, faibles à Meissonnier et à la cité-jardin, plus élevés à Villetaneuse, devenus incontrôlables à Pierrefitte. On notera également qu'au sein du logement social, le patrimoine le plus ancien est mieux reconnu (le label Patrimoine $\mathrm{XX}^{\mathrm{e}}$ siècle cible la période 1945-1975, excluant les cas des Poètes et de Renaudie), tandis que la reconnaissance de l'architecture moderne et des grands ensembles (Amougon, 2006; Pouvreau, 2011) constitue des problèmes spécifiques.

D'autres comparaisons seraient possibles, au sein de Plaine Commune (la Maladrerie à Aubervilliers a par exemple été labellisée Patrimoine du XXe siècle), du 93 (avec la cité de l'Etoile à Bobigny ou les Courtillières à Pantin, deux cas qui ont connu une publicité nationale, au même titre que la cité des Poètes), pour approfondir la piste d'un troisième type de patrimonialisation, par expertise. Si l'on s'appuie sur une étude à l'échelle nationale, le diagnostic d'une fréquente absence de mobilisation patrimoniale des habitants des quartiers populaires semble généralisable (Veschambre, 2013). Du fait $\mathrm{du}$ « désajustement entre regard expert et regard profane » (Heinich, op. cit., p. 76), elle ne survient que dans des cas exceptionnels, comme la cité Meissonnier ou l'Unité Le Corbusier de Firminy (Veschambre, 2000, 2010). Si le logement social peine paradoxalement à être le patrimoine des habitants, il peut être construit comme patrimoine pour mieux habiter. À l'épreuve de la crise, le patrimoine du logement social oscille entre victime expiatoire et remède à consolider.

\section{BIBLIOGRAPHY}

Albecker M.-F., 2014. Recycler les premières couronnes des villes globales : politiques d'aménagement urbain et restructurations des banlieues industrielles de Paris et New York. Thèse de l'Université Paris 1 Panthéon-Sorbonne.

EchoGéo, 33 | 2015 
Association Toit et moi, 2015. Les HLM. Une aventure collective. Saint-Denis, Éditions PSD, 143 p.

Aguilard Y., 1982. La Chartreuse de Mirande. Le monument historique, produit d'un classement de classe, Actes de la recherche en sciences sociales $n^{\circ} 42$, p. 76-85.

Amougou E., 2006. Les grands ensembles : un patrimoine paradoxal. Paris, L'Harmattan, 175 p.

Auduc A. (dir.), 2012. Ces patrimoines qui font territoire. Actes du colloque régional 24-25 novembre 2011 Région Ile de France. Paris, Somogy, 246 p.

Bacqué M.-H., Fol S., 1997. Le devenir des banlieues rouges. Paris, L'Harmattan, 215 p.

Bacqué M.-H., Sintomer Y., 2001. Affiliations et désaffiliations en banlieue. Réflexions à partir des exemples de Saint-Denis et d'Aubervilliers. Revue française de sociologie, 42-2, p. 217-249.

Bellanger E., Mischi J. (dir.), 2013. Les territoires du communisme. Élus locaux, politiques publiques et sociabilités militantes. Paris, A. Colin, $302 \mathrm{p}$.

Castel R., 1991. De l'indigence à l'exclusion, la désaffiliation. In Donzelot J. \& al., Face à l'exclusion, le modèle français. Paris, Éditions Esprit, p. 137-168.

Castel R. 1995. Les Métamorphoses de la question sociale. Une chronique du salariat. Paris, Fayard, 93 p.

Castel R. 2009. Individus par excès, individus par défaut. In Corcuff P., Le Bart C., De Singly F., L'individu aujourd'hui. Rennes, Presses Universitaires de Rennes, p. 293-306.

Chenevez A., 2004. From Tony Garnier to the Urban Museum: the birth of a cultural housing project. Museum International, Volume 56, Issue 3, p. 77-84.

Donzelot J., 2012. A quoi sert la rénovation urbaine ? Paris, PUF, collection La ville en débat, 237 p.

Donzelot J., Jaillet M.-C., 1999. La nouvelle question urbaine. Esprit, nº 258, p. 87-114.

Douay N., 2012. L'activisme urbain à Montréal : des luttes urbaines à la revendication d'une ville artistique, durable et collaborative. L'Information géographique, vol. 76, p. 83-96.

Guillerm E., 2006. La réception de la ZAC des Poètes à Pierrefitte. Mémoire de M1 de l'Université Paris 1 Panthéon-Sorbonne sous la direction de C. Loupiac, 181 p.

Heinich N., 2009. La fabrique du patrimoine : de la cathédrale à la petite cuillère. Paris, Éditions de la $\mathrm{MSH}, 286 \mathrm{p}$.

Jacquot S., Gravari-Barbas M., Fagnoni E., 2013. Patrimonialisation et tourisme dans la région métropolitaine parisienne. Le patrimoine, clé de métropolité touristique ? In Gravari-Barbas M., Fagnoni E. (dir), Métropolisation et tourisme. Comment le tourisme redessine la métropole parisienne. Paris, Belin, p. 103-117.

Lapeyronnie D., 2008. Ghetto urbain. Ségrégation, violence, pauvreté dans la France actuelle. Paris, R. Laffont, $624 \mathrm{p}$.

Pouvreau B., 2011. Faut-il « patrimonialiser » les grands ensembles? Métropolitiques. Consulté le 23/01/2012, http://www.metropolitiques.eu/Faut-il-patrimonialiser-les-grands.html

Pouvreau B., 2003. Le logement social en Seine-Saint-Denis. Paris, Itinéraires du patrimoine, $64 \mathrm{p}$.

Pouvreau B., 2008. Le Coin du feu à Saint-Denis (1894-1914). Une société coopérative d'HBM pionnière pour la Caisse des dépôts et consignations. Histoire urbaine ${ }^{\circ}$ 23, p. 41-54.

Prévot M., Douay N. (dir.), 2012. Activisme urbain : art, architecture et espace public. L'Information géographique, vol. 76, 100 p.

Rautenberg M., 2003. La rupture patrimoniale. Paris, Bernin, À la croisée, 173 p. 
Semmoud N., 2005. Valorisation patrimoniale et changement social : un pléonasme? In GravariBarbas M. (dir.), Habiter le patrimoine : enjeux - approches - discours. Rennes, Presses universitaires de Rennes, p. 265-280.

Veschambre V., 2000. Patrimonialisation et enjeux politiques : les édifices Le Corbusier à Firminy. Norois, $\mathrm{n}^{\circ} 185$, Patrimoine et environnement : les territoires du conflit, tome 47, p. 125-138.

Veschambre V., 2010. Comment concilier patrimonialisation ambitieuse et rénovation urbaine dans un grand ensemble? Le cas de Firminy-Vert. In Harismendy P. (dir.), Rénovation urbaine et patrimoine. Ville de Saint-Brieuc, p. 121-132.

Veschambre V., 2013. Défense de l'habitat social versus « patrimoine » : deux registres d'argumentation contre les démolitions qui ne convergent pas. In Desponds D. (dir.), Les habitants, acteurs de la rénovation urbaine? Rennes, PUR, p. 46-61.

Veschambre V., 2014. Les grands ensembles français : un patrimoine encombrant en ce début de XXIe siècle. In Djament-Tran, G., San Marco, P. (dir.), La métropolisation de la culture et du patrimoine. Paris, Éditions Le Manuscrit, collection Fronts pionniers, p. 367-406.

Wacquant L., 2006. Parias urbains. Ghetto - banlieues - État. Paris, La Découverte, 331 p.

\section{NOTES}

1. Même si la cité 212 Germain Dorel du Blanc Mesnil a été inscrite à l'inventaire supplémentaire des Monuments Historiques en 1996, en réaction à un projet de démolition partielle du bailleur.

2. Francis Chassel, Pierre Quercy, Rapport sur le devenir des logements construits à Villetaneuse par Jean Renaudie, p. 9-10 : www.culture.gouv.fr/culture/.../rapports/VilletaneuseRapport05.pdf

3. L'Amicale des locataires réunie autour de la future maire communiste se mobilise contre les malfaçons et l'insécurité et promeut la convivialité, organisant par exemple une fête de quartier annuelle

4. Un taux de chômage de $33 \%$, une taille moyenne des ménages de 3,6 personnes, une population composée à $44 \%$ de moins de 20 ans et de $32 \%$ de personnes étrangère selon le recensement de 1999.

5. Serge Renaudie signe la pétition, Renée Gailhoustet mène la mobilisation avec Jean-Pierre Lefebvre, ancien directeur de la SODEDAT 93.

6. J. Nouvel se mobilise par exemple. Cf. lettre du 11 novembre 2009 reproduite sur le site de Docomomo : http://www2.archi.fr/DOCOMOMO-FR/dossier-poetes-pierrefitte.htm.

7. Sources: Vivre à Pierrefitte, mars 2011, p. 19 et Le Parisien: http://www.leparisien.fr/ montreuil-93100/partiellement-demoli-le-quartier-fantome-attend-13-02-2010-814271.php

8. Source : Vivre à Pierrefitte, février 2010, p. 6.

9. Caroline Taix, «Architectes contre résidents : la démolition d'une cité du 93 fait débat », Le Point, 01/02/2010: http://www.lepoint.fr/actualites-societe/2010-02-01/architectes-contreresidents-la-demolition-d-une-cite-du-93-fait/920/0/419633

10. Source : http://www.pss-archi.eu/immeubles/FR-93059-26106.html.

11. La décision du tribunal considère que la Cité des Poètes "n'a pas atteint les objectifs poursuivis par son concepteur en matière d'écologie et de qualité de vie de ses habitants" et "ne peut être regardée comme un symbole d'une période de l'histoire de l'architecture, qui ferait partie d'un patrimoine à protéger ou à mettre en valeur" Source : tf1 et AFP : http://lci.tf1.fr/ france/justice/2010-02/la-cite-des-poetes-va-etre-demolie-5710738.html.

12. en majorité relogés hors du quartier, et en partie remplacés par des accédants à la propriété, leur mémoire a été - difficilement - collectée dans un DVD, 
13. «nous on est pas dans une réserve indienne, qu'est-ce que vous venez voir ? C'est ça c'est les pauvres que vous venez voir?».

14. Il s'agit d'une « discipline artistique (...) issue de la culture urbaine qui consiste à prendre en photographie diverses sources lumineuses en mouvement à l'aide d'un appareil stabilisé et réglé sur un long temps d'exposition afin de produire des traits de lumières sur les clichés » (source : Office du tourisme de Plaine Commune Grand Paris : http://www.saint-denis-tourisme.com/cited-etoiles/st-denis/tabid/14118/offreid/004f20d1-220a-44f3-b1ab-2f510f3cf66e/detail.aspx).

15. Qui a accueilli 5300 personnes entre 2008 et 2013, pour moitié Stanois, pour moitié extérieurs.

\section{ABSTRACTS}

At a time of crisis in metropolitan housing for ordinary people, this article uses the case of Plaine Commune to show that mobilizing people to promote the heritage status of social housing remains exceptional ("cité Meissonier" in Saint-Denis). Rather, the promotion of heritage is based on the views of experts ("cité Renaudie" at Villetaneuse), whilst intercommunality promotes residential activism (as in the garden-suburb of Stains). Promotion of heritage is threatened in two ways: mobilization against the notion by residents experiencing terrible social problems ("Cité des Poètes" in Pierrefitte), or the departure of such people away from social housing.

À l'heure d'une crise métropolitaine de l'habitat populaire, cet article montre à partir du cas de Plaine Commune que la mobilisation des habitants en faveur de la patrimonialisation du logement social reste exceptionnelle (cas de la cité Meissonnier de Saint-Denis), tandis que domine une patrimonialisation par expertise (cas de la cité Renaudie de Villetaneuse) et que l'intercommunalité promeut un militantisme de l'habiter (cas de la cité-jardin de Stains). Cette patrimonialisation est tendue entre deux risques : la mobilisation anti-patrimoniale d'habitants confrontés à un seuil rédhibitoire de problèmes sociaux (cas de la cité des Poètes à Pierrefitte) et la sortie du logement social.

\section{INDEX}

Mots-clés: banlieue, conflit, logement social, patrimonialisation, Plaine Commune

Keywords: suburbs, conflict, heritage, Plaine Commune, social housing

Subjects: Sur le Champ - Sur le Terrain

\section{AUTHOR}

\section{GÉRALDINE DJAMENT-TRAN}

Géraldine Djament-Tran, djament@unistra.fr, est Maître de conférences en géographie à l'Université de Strasbourg, membre de l'UMR Sociétés Gouvernements et Acteurs en Europe, membre associée à l'Equipe interdisciplinaire de recherches sur le tourisme de l'Université Paris 1 Panthéon-Sorbonne. Elle a publié récemment : 
- Djament-Tran G., San Marco P. (dir.), 2014. La métropolisation de la culture et du patrimoine. Paris, éditions Le Manuscrit, collection Fronts pionniers, $442 \mathrm{p}$.

- Djament-Tran G., 2013. La patrimonialisation dans les quartiers péricentraux de la « ville créative ", entre marketing et recyclage métropolitain. Étude comparée de Plaine Commune et du quartier Ostiense à Rome. In Fagnoni E. (coord.). La ressource territoriale entre patrimoine et création. Bulletin de l'Association de Géographes français, 2013-2, p. 153-169.

- Djament-Tran G., 2012. Habiter les périphéries du centre historique : le cas du quartier Ostiense à Rome, entre métropolisation et patrimonialisation. In Vallat C., Lemarchand N., Lesourd M.

(coord.), Façons d'habiter les territoires de la ville aujourd'hui. Historiens et Géographes, ${ }^{\circ}$ 420, p. 105-110. 